\title{
Stability of Signal Transformation Method for Triangular Waveform Tracking
}

\author{
Ali Bazaei, S.O. Reza Moheimani, and Abu Sebastian
}

\begin{abstract}
This paper is concerned with the mathematical analysis of signal transformation approach to triangular waveform tracking. We provide necessary and almost sufficient conditions for stability and convergence of tracking error for a general class of plants and compensators. Simulation results are presented that reveal the conditions under which the incorporation of signal transformation blocks in a linear feedback loop may introduce control performance improvements.
\end{abstract}

\section{INTRODUCTION}

Accurate tracking of a fast triangular waveform is one of the major challenges in scanning probe microscopy [1], [2] and other scanner-based devices such as optical scanners and selective laser sintering (SLS) machines [3], [4]. Raster scanning is also used in emerging probe-based data storage devices that demand high speed positioning with limited control bandwidth, due to measurement noise, actuator limitations, etc. [5], [6]. The triangular waveform contains all odd harmonics of the fundamental frequency. The high-frequency content in a fast triangular waveform can excite the resonant modes of the plant and subsequently degrade the tracking performance.

In [7], the concept of signal transformation was put forward as a novel method to track triangular waveforms in a nanopositioning system. The method showed significant closed-loop performance improvement compared with the ordinary one degree-of-freedom (1-DoF) feedback-controlsystem $^{1}$ having a similar control bandwidth [7]. However, only a second-order plant with no zeros and a doubleintegrator compensator were considered in [7] without any stability analysis.

In this paper, we provide an analysis of the signal transformation method, which leads to sufficient and almost necessary conditions for stability of the control system when tracking a triangular waveform. As in [7], the plant will be assumed to have a unity low-frequency gain. This condition is not so restricting as long as a stable feedback loop with an integrating compensator can be wrapped around the original plant. Furthermore, the analysis in this paper includes plants and compensators that have strictly proper transfer functions with arbitrary poles and zeros. The remainder of this paper is organized as follows. The signal transformation method

A. Bazaei and S.O.R. Moheimani are with School of Electrical Engineering and Computer Science, The University of Newcastle Australia, Callaghan, NSW 2308. Ali. Bazaei@newcastle.edu.au, Reza.Moheimani@newcastle.edu.au

A. Sebastian is with IBM Zürich Research Laboratory, CH-8803 Rüschlikon, Switzerland. a se@zurich.ibm. com

${ }^{1}$ In this paper, the $1-\mathrm{DoF}$ feedback control system will be referred as ordinary feedback system is briefly reviewed in section II. Necessary and sufficient conditions for exponential stability of the system are formulated in section III. Steady-state behavior as well as sufficient and almost necessary conditions for convergence of tracking error to bounded limits are quantified in Section III-A. In Section IV, empirical conditions are presented under which the control performance is improved after incorporation of the signal transformation in the ordinary feedback system.

\section{SignAL TRANSFORMATION}

Signal transformation method incorporates appropriate mappings between non-smooth signals (e.g. triangular waveforms) and smooth signals (e.g. ramps) in a control system to improve the tracking error while keeping the closed-loop bandwidth low to limit the projected measurement noise [7]. The signal transformation method for control of a SISO plant is described by the hybrid control system shown in Figure 1, where $\Phi$ and $\Phi^{-1}$ refer to the signal transformation mappings, which in the case of triangular signal tracking use piecewise constant gains $g_{1}$ and $g_{2}$, as well as biases $b_{1}$ and $b_{2}$, that can be presented in the following forms,

$$
g_{1}=g_{2}=(-1)^{i-1}, b_{2}=2 a_{0} \text { floor }(i / 2), b_{1}=(-1)^{i} b_{2}
$$

Here, $a_{0}$ is the amplitude of the desired triangular waveform $x_{d}$, which has period $2 T$, as shown in the left top insert in Figure 1, and $i$ is the index of half period defined as:

$$
i(t)=k, \text { if } t \in[k T-T, k T), k=1,2,3, \ldots
$$

The signal transformation blocks, which use $g_{2}$ and $b_{2}$, can convert the non-smooth periodic triangular signal $x_{d}$ to a smooth ramp signal denoted by $r$ in the right top insert in Figure 1. The signal transformation block between the plant and compensator does the reverse action, i.e. it can convert the smooth ramp signal into the non-smooth triangular signal. Under the ideal circumstances, where the plant is a unity gain transfer function and its output is perfectly following the desired signal, the input/output signals at compensator block will be smooth signals with no breaks or discontinuities and the burden of providing appropriate non-smooth trajectories at the actuator, which demands a high control bandwidth in an ordinary feedback system, is done by the signal transformation blocks. In this way, the compensator can be designed with a smaller closed-loop bandwidth in favor of rejecting the measurement noise without deteriorating the steady-state error. 


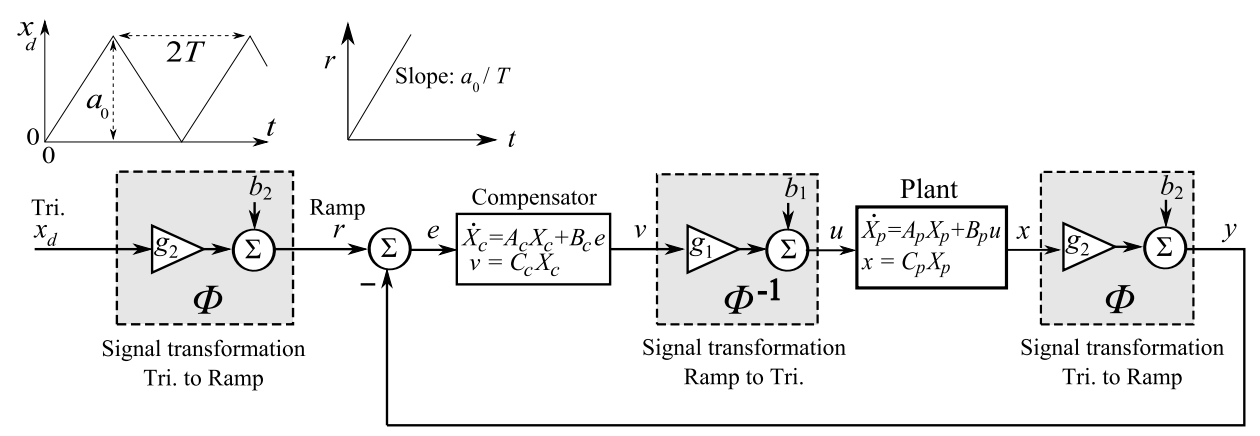

Fig. 1. Schematic diagram of signal transformation method for triangular waveform tracking.

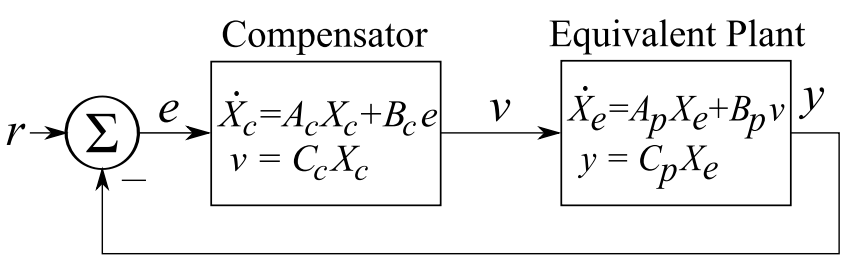

Fig. 2. Schematic diagram of the equivalent control system.

\section{StABILITY ANALYSIS}

We assume that the plant and compensator dynamics are of degrees $n_{p}$ and $n_{c}$ and are described by linear-time-invariant state-space matrix sets $\left[A_{p}, B_{p}, C_{p}\right]$ and $\left[A_{c}, B_{c}, C_{c}\right]$ with $X_{p}$ and $X_{c}$ referring to the corresponding state vectors, respectively. The feedthrough matrices have been assumed zero. To start the analysis we merge the plant and its adjacent signal transformation blocks into a unified state-space model, called equivalent plant. Hence, we wish to determine under what circumstances, the simple control system shown in Figure 2 is equivalent to the original hybrid control system in Figure 1, i.e. with the same ramp signal $r(t)$ in both control systems, the time histories of variables $e, v, X_{c}$, and $y$ in the equivalent system shown in Figure 2 are the same as the corresponding variables in the original system shown in Figure 1. The following lemma provides the conditions for the foregoing equivalence.

Lemma 3.1: In a time interval $t \in(i T-T, i T)$, the hybrid control system in Figure 1 is equivalent to the control system in Figure 2 and state $X_{e}$ of the equivalent plant is related to the plant state by equations

$$
X_{e}:=\frac{1}{g_{1}}\left(X_{p}+F\right), F:=A_{p}^{-1} B_{p} b_{1},
$$

provided that the gains and biases are constants (in the time interval) satisfying the following relationships

$$
g_{1} g_{2}=1, b_{2}-g_{2} C_{p} A_{p}^{-1} B_{p} b_{1}=0,
$$

and the equivalent state vector at the start of the time interval is initialized according to (3).

Conditions (4) are satisfied with the selected gains and biases in Equations (1) if the plant has a unity DC gain $\left(\delta_{0}=1\right)$. If the plant has a transfer function of the form:

$$
P_{o l}(s):=\frac{x(s)}{u(s)}=\frac{\delta_{0}+\delta_{1} s+\cdots+\delta_{n_{p}-1} s^{n_{p}-1}}{1+\epsilon_{1} s+\cdots+\epsilon_{n_{p}} s^{n_{p}}},
$$

its state-space realization can be written by the following canonical form:

$$
\begin{aligned}
& A_{p}=\left[0_{\left(n_{p}-1\right) \times 1}, I_{n_{p}-1} ; \frac{-1}{\epsilon_{n_{p}}}, \frac{-\epsilon_{1}}{\epsilon_{n_{p}}}, \cdots, \frac{\epsilon_{n_{p}-1}}{-\epsilon_{n_{p}}}\right], \\
& B_{p}=\left[0_{\left(n_{p}-1\right) \times 1} ; \frac{1}{\epsilon_{n_{p}}}\right], C_{p}=\left[\delta_{0}, \cdots, \delta_{n_{p}-1}\right] .
\end{aligned}
$$

The overall state vector $X$ of the equivalent closed loop system, defined as:

$$
X:=\left[\begin{array}{c}
X_{e} \\
X_{c}
\end{array}\right],
$$

obeys the following state-space equation

$$
\left\{\begin{array}{l}
\dot{X}=A X+B r \\
y=C X
\end{array}\right.
$$

where

$$
\begin{gathered}
A:=\left[\begin{array}{cc}
A_{p} & B_{p} C_{c} \\
-B_{c} C_{p} & A_{c}
\end{array}\right], B:=\left[\begin{array}{c}
0_{n_{p} \times 1} \\
B_{c}
\end{array}\right], \\
C:=\left[\begin{array}{ll}
C_{p} & 0_{1 \times n_{c}}
\end{array}\right]
\end{gathered}
$$

The equivalent plant state $X_{e}$ must be initialized by (3) at the start of each half period, which requires knowledge of plant state $X_{p}$. To use the equivalent control system as a stand-alone machinery for analysis, appropriate formulas are necessary to update the equivalent state at the switching moments $t=i T$. The following theorem gives the updating relationships at the switching moments.

Theorem 3.2: With the triangular reference signal shown in Figure 1, signal transformation parameters (1), and unity DC gain for the plant, the overall state vector of the equivalent control system just before a switching moment obeys the recursive formula:

$$
\begin{gathered}
X_{1}^{-}:=X\left(T^{-}\right)=E X_{0}+H \\
X_{i+1}^{-}:=\left.X(t)\right|_{t=(i+1) T^{-}}=\hat{A} X_{i}^{-}+J i+H, i=1,2,3, \ldots,
\end{gathered}
$$

and the state just after a switching moment is updated using its value just before the switching moment as:

$$
X_{i}^{+}:=X\left(i T^{+}\right)=\hat{I} X_{i}^{-}+L i, i=1,2,3, \ldots,
$$

where $L$ is the constant $\left(n_{p}+n_{c}\right) \times 1$ vector:

$$
L:=\left[2 a_{0}, 0, \ldots, 0\right]^{T},
$$


and

$$
\begin{gathered}
\hat{I}:=\left[\begin{array}{cc}
-I_{n_{p}} & 0 \\
0 & I_{n_{c}}
\end{array}\right], X_{0}:=\left[\begin{array}{c}
X_{p}(0) \\
X_{c}(0)
\end{array}\right], \\
E:=e^{A T}, H:=\left[\frac{1}{T}(E-I) A^{-1}-I\right] A^{-1} B a_{0}, \\
\hat{A}:=E \hat{I}, J:=(E-I) A^{-1} B a_{0}+E L .
\end{gathered}
$$

Proof: For brevity, a sketch of the proof is presented here. In the original control system shown in Figure 1, the gains and biases have discontinuous changes at the switching times $t=i T(i=1,2,3, \ldots)$, which makes the signals $y$ and $u$ discontinuous. However, the states and outputs of the plant and compensator $\left(X_{c}, X_{p}, v, x\right)$ are continuous due to zero feedthrough matrices and the inherent integration actions in the compensator and plant state equations. Hence, the equivalent plant state $X_{e}$ has discontinuities at the switching times because of $g_{1}$ and $b_{1}$ (see Equation (3)). Thus, to maintain the equivalence of the simple control system shown in Figure 2 with the original control system over time intervals longer than a half period, we have to intentionally incorporate appropriate jumps in the equivalent plant state $X_{e}$ at each switching time. Using the commutativity of multiplication of $A^{-1}$ and $e^{A t}$, the solution of the state $X$ from state-equation (8) in the time interval $t \in(i T, i T+T)$ with ramp input $r=\frac{a_{0} t}{T}$, in terms of the state just after the switching moment $t=i T$, can be written in the following form.

$X(t)=e^{A t^{\prime}} X_{i}^{+}+\left[\left(e^{A t^{\prime}}-I\right)\left(a_{0} i I+A^{-1} \alpha\right)-\alpha t^{\prime} I\right] A^{-1} B$,

where

$$
t^{\prime}:=t-i T, \alpha:=\frac{a_{0}}{T}, X_{i}^{+}:=X\left(i T^{+}\right) .
$$

Equation (12) defines a discrete-time LTI dynamic system with $\hat{A}$ as the state matrix, $[J, H]$ as the input matrix, and $[i, 1]^{T}$ as the input vector whose first element is a discretetime ramp signal. Hence, a necessary condition for the closed-loop system to be free from exponentially unstable modes is that all eigenvalues of $\hat{A}$ are inside the unit disk. This condition is also a sufficient one because the state at the arbitrary time $t=i T+t^{\prime}$ depends on $X_{i}^{-}$through Equations (13) and (18) and variable $t^{\prime}$ is limited to $t^{\prime} \in(0, T)$, which shows that if $X_{i}^{-}$does not have any exponentially unstable mode, neither does $X(t)$. In the more general case, where the desired signal $x_{d}$ is an arbitrary bounded signal but the signal transformation parameters are kept as before with unity DC gain for the plant, Equation (13) will not change but Equations (18), (11), and (12) can be represented in the following forms:

$$
\begin{gathered}
X\left(t^{\prime}+i T\right)=e^{A t^{\prime}} X_{i}^{+}+\left(e^{A t^{\prime}}-I\right) A^{-1} B a_{0} i+W\left(i, t^{\prime}\right), \\
X_{1}^{-}=E X_{0}+W(0, T), \\
X_{i+1}^{-}=\hat{A} X_{i}^{-}+J i+W(i, T), i=1,2,3, \ldots,
\end{gathered}
$$

where

$$
\begin{aligned}
W\left(i, t^{\prime}\right)= & \left(e^{A t^{\prime}}-I\right) A^{-1} B a_{0} \frac{1-(-1)^{i}}{2}+ \\
& \int_{0}^{t^{\prime}} e^{A\left(t^{\prime}-\tau\right)} x_{d}(\tau+i T) d \tau B(-1)^{i}
\end{aligned}
$$

Since vector $W\left(i, t^{\prime}\right)$ is bounded, because of the boundedness of $x_{d}$, the aforementioned condition about the absence of exponentially unstable modes is not restricted to the triangular waveform and is also valid for arbitrarily bounded reference inputs.

Corollary 3.3: Assuming unity DC gain for the plant and signal transformation parameters (1), the hybrid control system is free from exponentially unstable modes if and only if the eigenvalues of matrix $\hat{A}$, defined in (17), are inside the unit circle.

\section{A. Steady-state behavior with triangular reference}

The signal transformation converts the original triangular reference into a ramp signal. However, it is not desirable for the plant states to grow linearly with time. The following theorem provides conditions under which the states of the plant remain bounded in steady-state conditions.

Theorem 3.4: Assuming the triangular reference input in Figure 1, unity DC gain for the plant, signal transformation parameters (1), and eigenvalues of matrix $\hat{A}$ inside the unit circle, the plant state in the hybrid control system will remain bounded if and only if either of the following conditions is satisfied.

$$
\begin{gathered}
\delta_{c}:=-C A^{-1} B=1 \\
P\left(t^{\prime}\right):=\left[I_{n_{p}}, 0\right] e^{A t^{\prime}} \hat{I}(I-\hat{A})^{-1} L+0.5 L=0, \forall t^{\prime} \in(0, T)
\end{gathered}
$$

Proof: For brevity, a sketch of the proof is presented here. Using equality $-A_{p}^{-1} B_{p}=[1,0, \ldots, 0]^{T}$ and successive use of Equation (12), the coefficient of $i$ in the steadystate solution of the plant state vector can be expressed in the following form:

$$
(-1)^{i} P\left(t^{\prime}\right)\left(\delta_{c}-1\right),
$$

which reveals that the plant state tends to a bounded value if and only if either the closed-loop DC gain $\delta_{c}$ is unity, or all the elements in the time-dependent vector $P\left(t^{\prime}\right)$, defined in (25), are identically zero.

Since condition (25) is almost impossible to occur, condition (24) is almost a necessary condition for bounded-ness of the plant state. The plant does not have any pole at the origin because of its unity DC gain. Hence, the only way for the closed-loop system to have a unity DC gain is that the compensator has at least one pole at the origin. Thus, a sufficient and almost necessary condition for bounded-ness of the plant state is that the compensator has at least one pole at the origin.

In the more general case of an arbitrary bounded reference signal $x_{d}$, using (22), the constant vector $H$ should be replaced by the bounded vector $W(i-k, T)$. In this way, all 
of the terms which grow with $i$, remain unchanged. Hence, the aforementioned condition about the bounded-ness of the plant state is not restricted to the triangular desired waveform and is valid for any arbitrary bounded reference signal $x_{d}$ as well.

Corollary 3.5: Assuming unity DC gain for the plant, signal transformation parameters (1), and eigenvalues of matrix $\hat{A}$ within the unit circle, a sufficient and almost necessary condition for the plant state to remain bounded in the hybrid control system is that the compensator has at least one pole at the origin.

The position error $e_{p}:=x_{d}-x$ is simply related to the tracking error $e:=r-y$ by the following formula.

$e_{p}:=x_{d}-x=g_{2}^{-1} e=(-1)^{i-1}(r-y)$, for $i T-T<t<i T$

Assuming the triangular desired signal shown in Figure 1 for $x_{d}$, the conditions mentioned in Corollary 3.5, condition (24), one can show that the profile of the error in steady-state can be expressed in the following form:

$$
\begin{array}{r}
\lim _{i \rightarrow \infty} e_{p}\left(t^{\prime}+i T\right)=(-1)^{i-1} C \\
{\left[e^{A t^{\prime}} \hat{I}(I-\hat{A})^{-1}(E-I)\right.} \\
\left.+e^{A t^{\prime}}-I\right] A^{-2} B \alpha
\end{array}
$$

where $t^{\prime} \in(0, T)$. Equation (28) can be further simplified into the following form if the compensator has at least two poles at the origin.

$\lim _{i \rightarrow \infty} e_{p}\left(t^{\prime}+i T\right)=(-1)^{i-1} C e^{A t^{\prime}}(\hat{I}-E)^{-1}(\hat{I}-I) A^{-2} B \alpha$

Equation (29) is useful for fast and accurate calculation of steady-state profile of the error. Moreover, for a first-order plant, vector $(\hat{I}-I) A^{-2} B$ is vanished and the steady-state error (29) is identically zero.

Remarks: The restriction of unity DC gain for plant may be removed if one can wrap an ordinary stable inner loop with integral action around the plant. In this case, the block named plant in Fig. 1 should contain the original plant and the inner loop. This method can also provide robustness against disturbances.

\section{Simulation Results}

To compare the control performance of the signal transformation method with that of an ordinary 1-DoF feedback system, a number of simulation results are presented in this section. To reduce effect of high frequency components of the measurement noise at the output, the controllers are designed such that the closed-loop transfer function from measurement noise $n$ to the plant output $x$ in the feedback system shown in Fig. 3 has a very low bandwidth. Such a constraint limits the tracking performance of the control system. In ordinary 1-DoF feedback control systems, for an acceptable tracking of a triangular reference, the fundamental frequency should be less than one third of the closed-loop bandwidth [8].

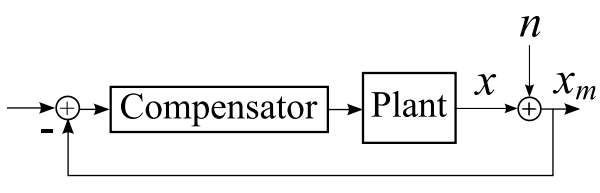

Fig. 3. Illustration of the ordinary 1-DoF feedback control system with measurement noise.

\section{A. Comparison of performances with no plant zero}

In this section, we consider two stable third order plants $P_{1}(s)$ and $P_{2}(s)$ with no zeros and poles in the following form.

$$
\begin{aligned}
& \text { Poles of } P_{1}=-100,-10^{3},-10^{4}(\mathrm{rad} / \mathrm{sec}) \\
& \text { Poles of } P_{2}=-70 \pm 70 i,-10^{4}(\mathrm{rad} / \mathrm{sec})
\end{aligned}
$$

The plants have unity DC gains and almost the same $-3 \mathrm{~dB}$ open-loop bandwidth of $16 \mathrm{~Hz}$. The dominant mode for the first plant is governed by just one pole and for the second plant by a pair of complex poles. It is pointed out that dynamics of plants $P_{1}$ and $P_{2}$ are quite similar to typical positioning systems with their dominant modes described by over-damped and under-damped mass-spring systems, respectively [9], [7]. The controller is a double integrator with the following transfer function, which is a popular controller for tracking of ramp signals [10].

$$
K(s):=\frac{k_{i}}{s}+\frac{k_{i i}}{s^{2}},
$$

The state-space representation of controller can be realized by the following matrices.

$$
A_{c}=\left[\begin{array}{cc}
0 & k_{i i} \\
0 & 0
\end{array}\right], B_{c}=\left[\begin{array}{c}
k_{i} \\
1
\end{array}\right], C_{c}=\left[\begin{array}{ll}
1 & 0
\end{array}\right]
$$

The desired triangular signal has unity amplitude $a_{0}=1$ and a half period of $T=1 \mathrm{sec}$. With unity controller gains $k_{i}=k_{i i}=1$ for both plants, the open-loop bandwidth of $16 \mathrm{~Hz}$ is reduced to the closed-loop bandwidth of $0.29 \mathrm{~Hz}$, which would significantly reduce the effect of measurement noise on the output (See Figure 4). Since the closed-loop bandwidth is less than the fundamental frequency of the triangular reference, the ordinary feedback system is not expected to have a good tracking performance. The control systems for the first and the second plant have almost the same phase margins of $+51^{\circ}$ with different gain margins of $60 \mathrm{~dB}$ and $42 \mathrm{~dB}$, respectively, and the following closed loop poles in $\mathrm{rad} / \mathrm{sec}$.

$$
\begin{aligned}
& \text { With } P_{1}=-0.5 \pm 0.87 i,-98.9,-10^{3},-10^{4} \\
& \text { With } P_{2}=-0.5 \pm 0.87 i,-69.5 \pm 69.5 i,-10^{4}
\end{aligned}
$$

The steady-state errors with signal transformation and with ordinary feedback system have the profiles shown in Figure 5(a) and (b), respectively. As expected, the ordinary feedback system cannot provide an acceptable performance at such a low bandwidth. With the proposed signal transformation, the tracking performances become acceptable and the maximum steady-state errors with the first and the second plants are 

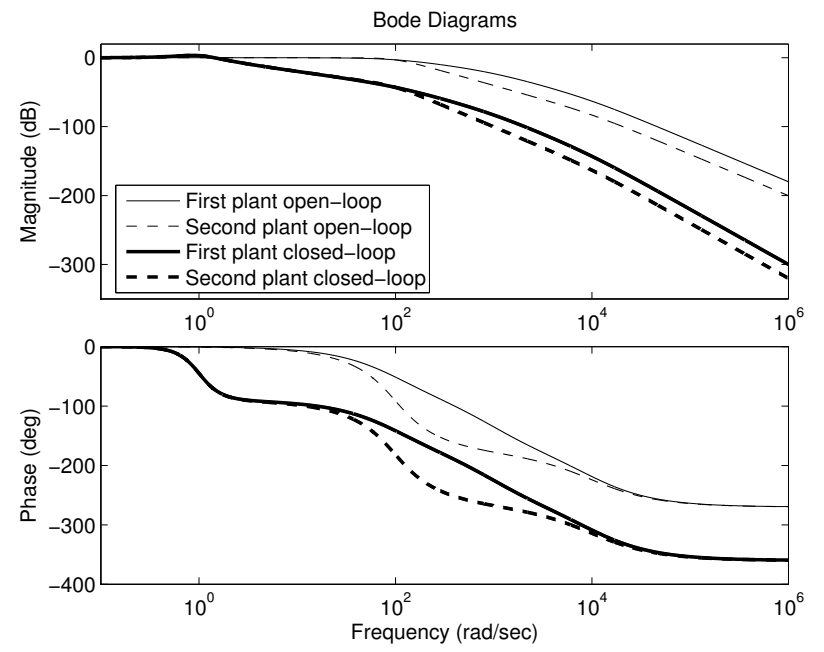

Fig. 4. Closed and open-loop Bode plots with no plant zeros.
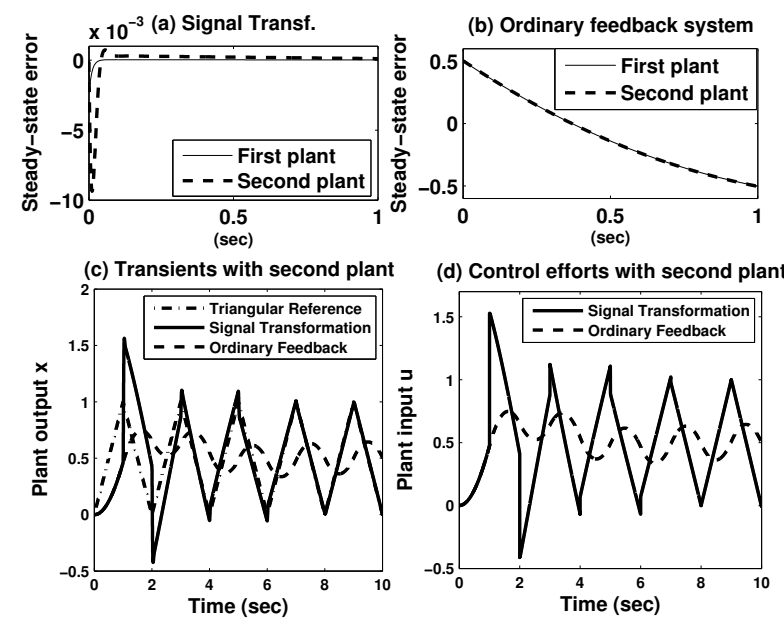

Fig. 5. (a) Steady-state error profiles in a half period with signal transformation (b) Steady-state error profiles in a half period in ordinary feedback system (c) Transient responses.

295 and 53 times better than that of the ordinary feedback system, respectively. The lower steady-state error associated with the first plant is due to first-order behavior of its dominant mode, which agrees with the conclusion mentioned at the end of Section III-A for the first-order plants. The following parameter can be considered as a measure of transient performance for the signal transformation method.

$$
\gamma:=\left|\lambda_{\max }(\hat{A})\right|
$$

This parameter represents the magnitude of the pole of the closed-loop discrete-time system (12), which is closer to the unit circle than the other poles and should be less than 1 for stability. Moreover, the smaller the value of $\gamma$, the faster is the decay rate of matrices $\hat{A}^{i-1}$ and $\hat{A}^{i}$ as $i$ tends to infinity. The value of this parameter with the selected parameter values is 0.613 for the first plant and 0.615 for the second one. The graphs of transient responses of the system with signal transformation and zero initial states are shown in
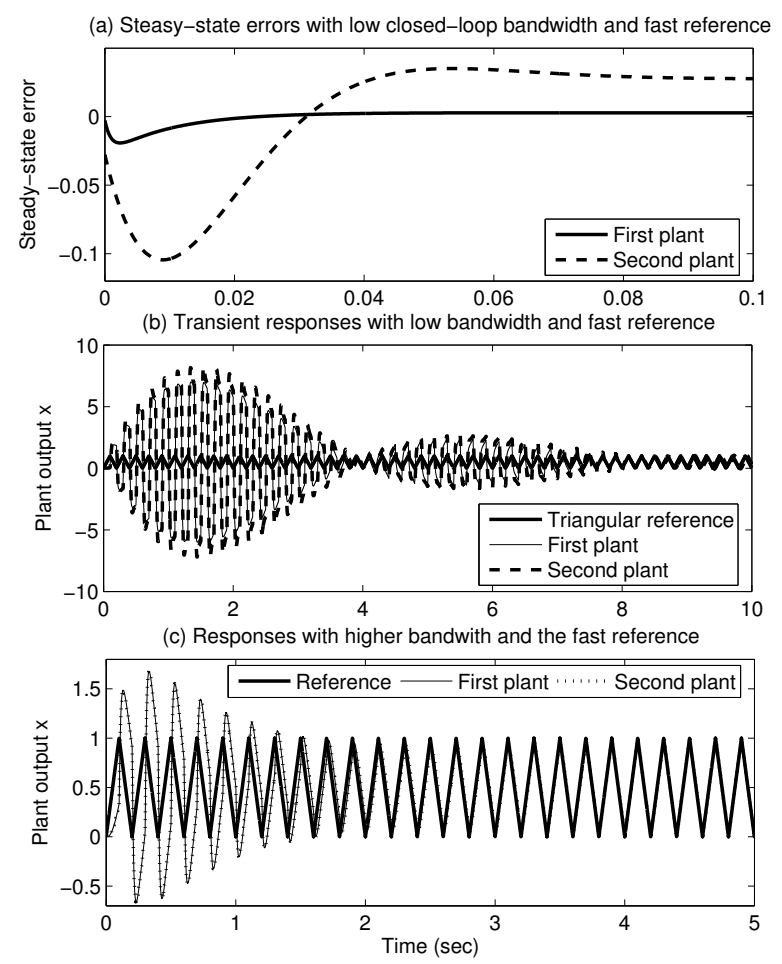

Fig. 6. Tracking of a $5 \mathrm{~Hz}$ triangular reference with signal transformation and low closed-loop bandwidth of $0.29 \mathrm{~Hz}$ (a) steady-state error profiles in a half period (b) Transient responses (c) Closed-loop responses with a more bandwidth of almost $2 \mathrm{~Hz}$ obtained by $k_{i}=k_{i i}=10$.

Figure 5(c). The mentioned results clearly show the overall improvement obtained by the signal transformation method in this example.

If we decrease the period of the desired triangular reference, the steady-state error will increase with the signal transformation, and so will $\gamma$, which renders the system unstable, while in the ordinary feedback system the stability is not affected by the period. In the above example, if we increase the fundamental frequency of the triangular reference to $5 \mathrm{~Hz}$ (16 times more then the closed-loop bandwidth) while keeping the other parameters unchanged, the first plant with signal transformation has a very low steady-state error as shown in Figure 6(a) with the thick solid line. However, the transient response with the signal transformation is deteriorated as the period of the triangular reference decreases and so is the settling time, as shown in Figures 5(c) and $6(b)$. With the fast triangular reference of $5 \mathrm{~Hz}$, the second plant with a second-order dominant mode exhibits more steady-state error compared with the first plant. Hence, if the transient in the signal transformation method can be tolerated, the frequency of the triangular reference should not be increased much higher than the closed-loop bandwidth for plants with dominant modes of second-order or higher. In this example, we observed that the transient responses of the signal transformation scheme can be improved without deteriorating the steady-state performances by increasing the compensator gains $k_{i}$ and $k_{i i}$ at the expense of increasing the closed-loop control bandwidth (see Figure 6(c) and its 

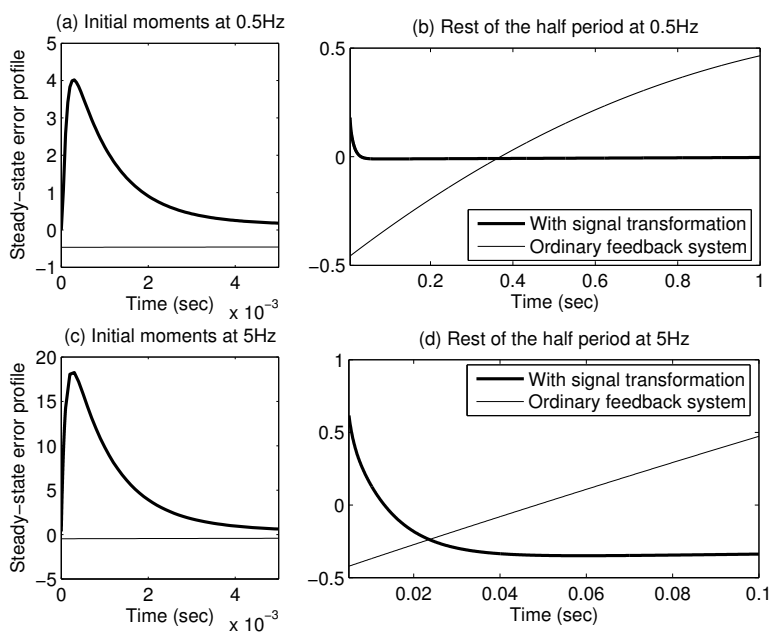

Fig. 7. Steady-state error profiles with signal transformation method as well as ordinary feedback system, unity integrator gains, unity amplitude triangular reference, and dominant zeros of -15 and $-150 \frac{\mathrm{rad}}{\mathrm{sec}}$ included in the first plant. (a),(b) For a $0.5 \mathrm{~Hz}$ reference. (c),(d) For a $5 \mathrm{~Hz}$ reference.

explanation).

\section{B. Effect of plant zeros}

Before finding a general criterion under which the control performance is improved by the signal transformation method, it is helpful to consider the effects of plant zeros by simulation, although plants with dominant zeros are not usual in positioning applications. If we allow for two zeros of -150 and $-1500 \mathrm{rad} / \mathrm{sec}$ in the first plant described in Section IV-A while keeping the plant's DC gain unity, we will still find the signal transformation method introducing similar improvements mentioned there. The improvements are also repeated even if either of the plant zeros has a sign change, which renders the plant a non-minimumphase system. However, if we move the plant zeros to -15 and $-150 \mathrm{rad} / \mathrm{sec}$, where the dominant zeros are closer to the origin than the dominant open-loop poles, the signal transformation method produces large errors at the initial moments of each half period, as shown in Figures 7(a) and 7(b) for a plant with the same poles as the first plant. In this case, where the closed-loop bandwidth is $0.27 \mathrm{~Hz}$ with unity compensator gains, the signal transformation method cannot improve the steady-state tracking performance of the ordinary feedback system for a faster triangular reference with fundamental frequency of $5 \mathrm{~Hz}$, as shown in Figures 7(c) and 7(d). In the foregoing case, the ordinary feedback system has an unlimited gain margin and a safe phase margin of $56^{\circ}$. However, its response to a unit step disturbance applied at the plant input has an undesirable overshoot of 35 , which is mainly due to the dominant zero at $-15 \frac{\mathrm{rad}}{\mathrm{sec}}$.

\section{CONCLUSIONS}

Signal transformation method can improve the steady-state error in tracking of a periodic triangular desired signal while keeping the closed-loop control bandwidth quite low for reduction of projected measurement noise. When the plant low-frequency-gain is unity and the signal transformation parameters are defined according to (1), the closed-loop system with signal transformation is free from exponentially unstable modes if the eigenvalues of matrix $\hat{A}$, defined in (17), are inside the unit circle. Moreover, a sufficient and an almost necessary condition for the plant state to remain bounded is that the compensator has at least one pole at the origin. Simulation shows that when the compensator is simply a linear combination of an integrator and a double integrator, as defined in (32), the signal transformation makes the control performance better in comparison with an ordinary $1-$ DoF feedback control system. This is true, provided that the dominant plant zeros have magnitudes larger than the dominant plant poles, the period of the reference signal is high enough in comparison with the settling-time of the open-loop plant, and the ordinary closed-loop system, which does not include any signal transformation, has acceptable performance characteristics, which means acceptable phase and gain margins and input disturbance response.

\section{ACKNOWLEDGMENTS}

The authors wish to thank IBM Zurich Research Labs and ARC Centre of Excellence for Complex Dynamic Systems and Control for their support of this work. Special thanks go to Evangelos Eleftheriou and Haris Pozidis for their support.

\section{REFERENCES}

[1] S. Devasia, E. Eleftheriou, and S. O. R. Moheimani, "A survey of control issues in nanopositioning," IEEE Transactions on Control Systems Technology, vol. 15, pp. 802-823, September 2007.

[2] I. A. Mahmood, S. O. R. Moheimani, and K. Liu, "Tracking control of a nanopositioner using complementary sensors," IEEE Transactions on Nanotechnology, vol. 8, pp. 55-65, January 2009.

[3] J.-Y. Yen, Y.-C. Yeh, Y.-H. Peng, and J.-F. Lee, "Application of the continuous no-reset switching iterative learning control on a novel optical scanning system," Mechatronics, vol. 19, no. 1, pp. 65-75, 2009

[4] D. Zhiqiang, Z. Zude, A. Wu, and C. Youping, "A linear drive system for the dynamic focus module of SLS machines," The International Journal of Advanced Manufacturing Technology, vol. 32, no. 11-12, pp. 1211-1217, 2007.

[5] H. Pozidis, P. Bachtold, J. Bonan, G. Cherubini, E. Eleftheriou, M. Despont, U. Drechsler, U. Durig, B. Gotsmann, W. Haberle, C. Hagleitner, D. Jubin, A. Knoll, M. Lantz, A. Pantazi, H. Rothuizen, A. Sebastian, R. Stutz, and D. W. Wiesmann, "Scanning probes entering data storage: From promise to reality," in IEEE Conference on Emerging Technologies - Nanoelectronics, pp. 39-44, Jan. 2006.

[6] A. Pantazi, A. Sebastian, G. Cherubini, M. Lantz, H. Pozidis, H. Rothuizen, and E. Eleftheriou, "Control of MEMS-Based ScanningProbe Data-Storage Devices," IEEE Transactions on Control Systems Technology, vol. 15, no. 5, pp. 824-841, 2007.

[7] A. Sebastian and S. O. R. Moheimani, "Signal transformation approach to fast nanopositioning," Review of Scientific Instruments, vol. 80, p. 076101, July 2009.

[8] S. Salapaka, A. Sebastian, J. P. Cleveland, and M. V. Salapaka, "High bandwidth nano-positioner: A robust control approach," Review of Scientific Instruments, vol. 73, no. 9, pp. 3232-3241, 2002.

[9] Y. Ting, C.-C. Li, H.-C. Jar, and C.-W. Chiang, "Design and control of a nano-precision cartesian platform," in IEEE/RSJ International Conference on Intelligent Robots and Systems, 2006.

[10] P. W. Belanger and W. L. Luyben, "Design of low-frequency compensators for improvement of plantwide regulatory performance," Industrial \& Engineering Chemistry Research, vol. 36, no. 5, pp. 53395347, 1997. 